\title{
Dysesthesia, CTCAE
}

National Cancer Institute

\section{Source}

National Cancer Institute. Dysesthesia, CT CAE. NCI Thesaurus. Code C143421.

A disorder characterized by distortion of sensory perception, resulting in an abnormal and unpleasant sensation. 